\title{
The Point: An Independent Resource Center for English Language Learners \\ Perrin Blackman
}

\section{The Butterfly Flaps Its Wings}

On Monday morning, October $22^{\text {nd }} 2012$, at 9:00 AM, two volunteers from the Applied English Center (AEC) made their way across the University of Kansas campus to help staff the four-week pilot program of our new independent resource center: The Point. They located the room, a bright open space on the top floor of the Anschutz Learning Studio, and set up their laptops in anticipation. For supplies, they had some pens, a sign-up sheet, a candy jar, and a few white-board markers. They made sure that an appointment book was delivered to the help desk, and they began to discuss the possibilities of the new space. At 11:00, having had no visitors, the volunteers packed up the supplies and went on with their day, making a mental note to talk-up The Point in their own classes.

Soon word got around, and a few students (like scout bees from a backyard hive) began to hover. Most arrived with essays or practice tests in hand, but a few just wandered in asking for help with "listening” or "reading." Those volunteers who happened to be staffing when a student arrived quickly became aware of the lack of physical resources for instructors. While we had intentionally drawn our students away from their usual resources, we had unintentionally been cast adrift without our own familiar books, handouts, or lesson plans.

This conundrum arose because The Point is not a traditional independent resource center. We have the privilege of scheduling large blocks of time in a beautiful sunny room, but since the room is used by others, we cannot keep any supplies in the room. This restriction was the first major challenge we had to face. As temporary coordinator, I was secretly pleased with this limitation because it meant that I didn't have to say 'no' to, or make room for, well-meant and possibly less-than-useful gifts. The initial restrictions could have been seen as unpleasant, but instead, they gave structure to our plans.

Anticipating this issue, we had set up a Blackboard site on which instructors could communicate and share materials digitally. Even though most of our instructors are capable of teaching any subject or level, we all have areas in which we are more comfortable teaching. The challenge was to provide a wide range of up-to-date materials that could be accessed immediately and understood easily by both instructors and students. Thus, within a few days of opening, instructors began adding more websites and materials organized by topic and level. Our classes are divided into Reading/Writing, Speaking/Listening, and Grammar, so each of these areas was represented. As time went by, we also developed sections for general websites, study skills, vocabulary, and games. Some basic ground rules emerged, and in an attempt to avoid cluttering up the site, we sub-divided each category into websites and original printable materials, making sure that a distinction was drawn from the beginning. All materials were added with the assumption that anyone could use them at any time. Instructions for how to post were listed, and everyone was encouraged to add to and use the site.

As students came into the center, an unofficial protocol also emerged. A student would sign-in and we would have a brief chat to find out what they wanted help with. If they did not bring a specific assignment, we looked on the Blackboard site for possible materials. After going through a few websites and exercises together, we would offer to send these sites and materials to the student via email so they could practice more at home. A basic form letter was ultimately drafted and posted on the Blackboard site for other instructors to use to follow up on student visits. This process alerted the instructors to which areas on the Blackboard site needed more materials.

The Point had now become an academic resource center not just for students but for ourselves. Instructors were now able to peruse the Blackboard site and find materials to use in their own classes. We always had two staffers in the room at any given time to ensure coverage, so teachers also utilized the space to meet with students, grade papers, and chat with one another about possible teaching methods and issues that arose in their own classes. Thus, The Point evolved into a place where instructors could collaborate virtually and face-to-face. 
As we had hoped, simply being in a new space created room for exploration, and again, it was not just our students who benefitted. Space is a valuable commodity at the AEC, so we were able to appreciate the treasure trove of resources we found. In fact, by the semester's end, our newly-hired activity coordinator had already reserved another room nearby for a book club slated to start in Spring 2013. Other instructors discovered that they could check out laptops rather than carry their own across campus.

Nearby resources in the Anschutz Learning Studio include:

- Two complete floors of comfortable, quiet study space

- Conference rooms and other group study rooms that can be reserved

- Wireless access and plug-ins for laptops

- KU Info (general information service), reference librarians, and laptop checkout facilities

- A snack bar and an area with lockers

- $\quad$ The KU Writing Center headquarters

Thus, while we (as instructors) were out of our element, we were forced to do exactly what we wanted our students to do: look around and access resources! Taking things down to the very basics opened up a world of possibilities. The process of creating our independent learning center had begun, but in order to see where it might go in the future, it's necessary to look back at the initial conditions.

\section{Initial Conditions}

The idea for The Point emerged from the following facts:

1. Part of the AEC's mission is to prepare our students linguistically, academically, and culturally for success at KU. However, we know that simply attending class is not sufficient if our students are to make adequate progress. Our students need to reach out actively to get the assistance they need.

2. A number of our students tend to come from educational systems that do not emphasize independent learning. A significant number of students do not ask questions in class, make use of instructors' office hours, or use library and campus resources.

3. Many of our students are not prepared for the rigor that our program requires. They may be the first in their family to attend college, so they may not understand how to arrange their time and materials to their best advantage. Others tend to fall back on survival skills that may have worked in the past but are inappropriate now. Some students may spend their time memorizing, plagiarizing, and working long but ineffective hours in isolation.

4. Our students often self-segregate and yet say that they don't have time or opportunity to practice their English. They tend to have their classes in the same area, eat in the same location, and socialize with the same people. Many see the idea of joining a campus organization as a waste of time and are not keen on being dislodged from their comfort zone.

With these issues in mind, the AEC jumped at the idea of setting up a space in Anschutz Library in large part because the location places our students in the middle of a hive of academic activity. In order to find our room, students have to pass through two floors filled with KU students studying, conferring, researching, and working. For our students, the simple process of finding the room is an activity that bridges the gap between the shelter of the Intensive English Program and academic life at KU.

Our first steps were small but decisive. We scheduled the room, enlisted volunteer staffers, sent out announcements to students, and provided instructions and referral forms to the AEC faculty. We created a sign-up sheet to see how many students visited The Point. On this sheet, we collected the student's name, the topic of interest, student level, and the time 
of the visit. We also set up an appointment book which the staff of the Anschutz Library graciously offered to keep track of.

Working without a budget was one more structural parameter that could have been seen as restrictive but allowed us the chance to start small. A few other supplies were collected: a cart on which to keep books, a small box of office supplies, and the aforementioned candy jar (which proved to be quite popular among the staffers.) We also asked instructors to bring their laptops along to their sessions so they could access the Blackboard site.

As time passed, a public identity began to develop. Students started talking about "The Point," and instructors began to develop an idea of who we were in relation to each other and to the university as a whole. To start, we reached out to other KU faculty and staff in the area. I met with Dr. Therese Thonus, director of the KU Writing Center to ask for advice and make sure we were not creating any issues with her work. She gave me a vast amount of information about how to keep records, provide services, and head off potential problems. We also connected with library administration and staff to develop a network of people who could help us best use the Anschutz Learning Center and other library services. It's important to mention that this was not noticed as an afterthought, that as the coordinator, I was very much aware of how we were developing a sense of who we were over time and how that identity reiterated itself and helped us to form into a cohesive entity.

Once the essential structure was set up, the challenge was (and is) to strike a balance between too much activity and too little. Initially, the biggest perturbation was that there was too little activity. Instructors grew weary of waiting for students to arrive and began to arrive late or leave early. They didn't appreciate the chance to stock up materials on the Blackboard site in preparation for student visits. Instead, the time was seen as a bit of a break or time to get some grading done.

On the other hand, a simple "rush hour" of two students could send the instructors into a bit of a spin depending on what the student wanted and what the instructor was able to produce. However, the situation generally leaned much more toward too little activity, so the next step was to encourage more students to visit. Many of them, we realized, had gotten and ignored the initial email announcement, so a more personal touch was required. We had created small half-page information forms for instructors to give to students, but these had been sent digitally, so a packet of printed and cut forms was placed in a central location for easy access. Several instructors, particularly those who were volunteering, made announcements in class or handed out the information sheets to individual students with specific notes explaining what the student should work on during their visit. As a consequence, more students began to use the center.

\section{A Narrative Emerges}

The pilot program lasted only four weeks. Fifteen volunteers spent a total of 80 hours helping students or simply waiting for them to arrive. We had one whole-class visit from a level one group, and 8 individual visitors who visited a total of 15 times. Students came from each level from low to high-intermediate. The topics included: essay planning and revision, grammar practice, listening for dates and numbers, and increasing reading speed.

The only complaint from students was that they wanted The Point to be open more hours. We staffed the center from 911 daily and discovered that most students came in on the half hour (having come from a class) and tended to leave at least 15 minutes before closing so they could get to another class. Since we were open for 2 hours in a row, this allowed them plenty of time to work between 9:30 and 10:45. Some instructors were only scheduled for hour-long shifts, so there was a minor issue when they had to hand-off their student to the other staffer. Responses were positive. One student stated, “I can’t believe he [another student] is paying a tutor when he can go to The Point for free!” After their visit, two students from the Level One class sent emails to their instructor asking for The Point to be open when they could attend. With this sort of reaction, it is easy to see how the project will gain momentum rapidly and why it is extremely important to be reliable and attentive to student needs if we are to succeed. In the future, we hope to be open between 10 and 2 to catch the greatest number and variety of students. To fill this need, it might be necessary to use student assistants in 
combination with seasoned academic instructors. This, of course, would require setting up a more detailed staff training protocol.

At the AEC, students progress to the next level based on their scores on our proficiency exam, which is given at the end of each semester. Grades are also a factor in their placement, so students strive to do well in both areas. If students do not make adequate progress, they are placed on probation at which point they must meet several requirements including attaining at least a "B" in each class and scoring high enough on the Proficiency Test to move to the next level.

For the eight students who came to The Point during this 4 week pilot period, the average overall class grade was A- with 5 out of 8 students receiving straight A's in all their classes. The average increase in Proficiency Test total scores was 83 points, on a 0-480 point scale where 480 represents a passing score of 160 in each skill (Reading/Writing, Speaking/Listening, Grammar for Communication). Two students improved more than 100 points overall, with an increase of 119 and 182. Of the 8 students, two had been on academic probation, both of whom made progress on the proficiency test, but did not test into a higher level in one or more classes.

While our short run cannot account for the progress of these students, it is clear that students who do succeed are making use of this resource. Ironically, I had considered The Point to be a place for the average student rather than those on the extreme ends of the academic spectrum, but judging from the demographics, it seems that students on the high and low ends were more motivated to take advantage of the center. In the future, we will consider ways to target average students, perhaps by providing specific workshops on topics of general interest such as vocabulary, listening activities, grammar practice, and writing skills.

After the pilot program, instructors were polled for their opinions. We asked three open-ended questions, and four instructors responded. Their concerns and comments are summarized in Table 1 below.

Table 1

Summary of Opinions of Point Instructors on The Point Operations

1. What can we do to make The Point run more smoothly?

- It's great to have a trial run this semester and everything seems to be running smoothly. The set-up, including the $\mathrm{Bb}$ site, is there. Now that everything is in place, it will be easy to put a lot of energy into strategic marketing to attract students. I trust that will all come together next semester.

- $\quad$ Providing a couple of laptops would be great.

- We should get some graded readers and novels for students to check out. It would also be good to have a few reference books available on the cart.

- It's probably not necessary to change much of anything yet. One more full semester with no big changes might give the students a better chance to utilize this great service.

2. What concerns or questions do you have about the future?

- It will be difficult to argue for a teaching percentage ... until the work load increases. If students could sign up in their classrooms, you might be able to attract more students.

- I could see my recommending a student to go there for some one-on-one pronunciation work, but am wondering if teachers in other skill areas feel comfortable helping students with that.

- How will the staffers and volunteers be organized in the future? Who will be in charge of ensuring that staffers are trained and held accountable for being on time and following (or giving feedback about) the emerging protocol?

- If teachers choose to/are assigned to work here, can there be a percentage paid? On the other hand, asking everyone to do their part, like one hour a week for four weeks, might be even better ---- get more people involved, might create better 'advertising' to the students, etc...

3. What cool, wonderful, creative things can we do in the future?

- You could advertise short workshops (30 minute to the Point workshops).

- We are always in need of a room for pronunciation tutorials. [These could be done at The Point.]

- Could we provide Kindles (or similar) loaded with digital reading material? What kind of digital reading material can we provide?

- Special activities could be planned to coincide with things going on in the classes, i.e....practice for an 
upcoming presentation.

- We could start mid-semester with some proficiency-exam-like practice...

- $\quad$ Perhaps the Ss would just get a kick out of a 'special event' atmosphere, and in reality it could be set up to where they even teach each other!!

As for the future, we are limited only by our imagination and energy. Currently, enthusiasm is high, so we should follow through decisively with a widely promoted schedule and the continued collection of quality digital materials. We should also follow up on the idea of having special workshops or events to bring students and instructors in. To encourage communication, we ought to get as many instructors as possible involved either through paid percentages or volunteer work. Already, The Point has developed an identity and a life of its own. Since the basic structure is in place, and the foundation seems to be solid, we can expect many students and instructors alike to make their mark on this emerging process. 